\title{
Liver Metastases Pattern in Rectal Cancer: A Real-World Analysis in the SEER Database
}

Yang $X^{1 *}$, Ren $H^{2 *}$, Yang $X^{1}$, Zhang $X^{1}$, Sun $Y^{1}$, Shao $Y^{1}$, Zhang $L^{1}, L^{2 i} H^{1}$ and $F_{u} J^{1}$

${ }^{1}$ Department of Radiation Oncology, Shanghai J iao Tong University Affiliated Sixth People's Hospital, Shanghai, China

${ }^{2}$ Department of Orthopedics, Shanghai Pudong Hospital, Fudan University, Pudong Medical Center, Shanghai, P.R China

*Corresponding author: Xiaojing Yang, Department of Radiation Oncology, Shanghai J iao Tong University Affiliated Sixth People's Hospital, No. 60o, Yishan Road, Shanghai, 200233, China

Hanru Ren, Department of Orthopedics, Shanghai Pudong Hospital, Fudan University, Pudong Medical Center, Shanghai 201300, P.R China

Received: May 18, 2021; Accepted: June 14, 2021; Published: June 21, 2021

\begin{abstract}
Background: The liver is a common metastatic site of colorectal cancer. Rectal cancer patients with organ metastases are more liable to show poor prognosis. The hazard and forecast elements of liver metastases are need to be estimated in rectal cancer patients.
\end{abstract}

Methods: The data of newly diagnosed patients of rectal cancer with liver metastases are evaluated according to Surveillance, Epidemiology, and End Results (SEER) program between 2010 and 2016. The Overall Survival (OS) for dierent subgroups are appraised by Kaplan-Meier analysis and log-rank tests. Univariate and multivariable logistic analysis and Cox regression are performed to evaluate predictors and elements of the presence of liver metastases in new diagnosis, respectively.

Results: There are a total of 6,662 (11.1\%) rectal cancer patients paired with liver metastases. Factors including age (below), gender (female), marital status (unmarried), race (black), advanced T or $\mathrm{N}$ classification, presence of bone or lung metastases, and the absence of surgical treatments are importantly related to the occurrence of liver metastases. The median survival for liver metastases rectal cancer patients was 16.0 months. Indicators referring to elder age, black race, unmarried status, presence of bone, brain or lung metastases, and the absence of surgical treatments all predicted worse survival.

Conclusion: The data of our research provide corresponding risks and prognostic elements for liver metastases rectal cancer patients, which offer a way to predict the occurrence of rectal cancer and guide prophylactic treatment in clinical settings.

Keywords: Rectal neoplasms; Neoplasm metastasis; SEER program

\section{Abbreviations}

SEER: Surveillance Epidemiology and End Results; OS: Overall Survival

\section{Background}

Rectal cancer is known as the eighth most general malignancy, which was diagnosed globally with the number of 704,376 bringing up 310,394 cases of mortality in 2018 [1]. A considerable number of rectal cancer patients have a poor prognosis due to metastatic progression [2]. The liver is a common metastatic site of colorectal cancer, where synchronous liver metastases were discovered in 15\% to $25 \%$ of record [3]. With the progress in medical technologies, the survival time of rectal cancer patients is prolonged, nonetheless, the risk of liver metastasis is increased accordingly [4].

Rectal cancer patients with organ metastases are more liable to show poor prognosis. Studies have shown that surgical resection of liver metastases colorectal cancer patients prolonged the median survival by 6.7 months compared with patients without surgery [5]. Research with a large sample size showed that the primary site of the tumor is a critical prognostic factor for metastatic colorectal cancer [6]. Conrad et al. reported an ideal 5-year Overall Survival (OS) for synchronous rectal liver metastasis patients by individualized treatment [4]. However, rectal cancer and colon cancer do not share the same biological identities and clinical performance $[7,8]$. Meanwhile, the hazard and forecast elements for patients with newly diagnosed rectal cancer and liver metastases have not been fully studied. Thence, in order to facilitate the early diagnosis of liver metastases rectal cancer patients and improve the survival rate of these patients, the characteristics of patients with rectal cancer liver metastases should be summarised.

The National Cancer Institute's Surveillance, Epidemiology and End Results (SEER) is an openly accessible database from the United States which provides cancer incidence and survival information. With the assistance of SEER data, this research was designed to assess the incidence proportion of liver metastases rectal cancer patients who diagnosed cancer at a population-based level. We also attempted to quantify survival estimates and to check predictors of poorer survival among patients with liver metastases manifest at diagnosis of rectal cancer.

\section{Methods}

\section{Cohort definition}

The data of patient were obtained from the SEER program database between 2010 and 2016. SEER ${ }^{\star}$ Stat software (Version 8.3.5, http://seer.cancer.gov/seerstat/download) was used to define rectal 
cancer patients with the inclusion criteria: (1) clear pathological diagnosis; (2) Diagnostic time between 2010 and 2016; (3) The "primary site" labelled as "C20.9-Rectum, NOS"; (4) TNM staging according to the seventh edition of AJCC; (5) Known survival time. An exclusion procedure was then performed to determine cases of rectal cancer with liver metastases. Cases with only autopsy or death certificates, invalid follow-up and unknown liver metastases were excluded. The thorough program is displayed in Figure 1.

\section{Parameters}

The demographic data involved sex (male, female), marital status (married, unmarried, unknown), age at diagnosis $(<65$, and $\geq 65$ years), and race [white, black, and other (American Indian/ Alaska Native or Asian/Pacific Islander)]. The clinical properties incorporated T stage (T0, T1, T2, T3, T4, and unknown), N stage (N0, $\mathrm{N} 1, \mathrm{~N} 2$, and unknown), other organ metastases include bone (none, yes, and unknown), brain (none, yes, and unknown), and lung (none, yes, and unknown). The treatment included the surgical condition (none, yes, and unknown) where the surgical cases were extracted by a combination of two variables in SEER database (RX SUMMSURG PRIM SITE and RX SUMM-SURG OTH REG/DIS). The data of survival status and time of each patient were included as well.

\section{Statistical analysis}

The risk factors for newly diagnosed rectal cancer patients with liver metastasis were primarily determined by univariate logistic regression. The detected major factors by univariate logistic regression $(\mathrm{P}<0.05)$ would be further dissected through multivariate logistic regression. OS is delimited as the time from diagnosis of rectal cancer to death, which denoted as the main consequence of the survival analysis. Differences in survival were dissected by Kaplan-Meier analysis and log-rank tests. Meanwhile, a multivariate Cox proportional hazard regression was carried out based on the above factors. In addition, differences between patients undergoing surgery and those without surgery for rectal cancer were detected by univariate and multivariate logistic regression. We use SPSS 23.0 (IBM Corporation, Armonk, NY) to execute statistical analyses. It is considered that $\mathrm{P}<0.05$ is statistically significant.

\section{Results}

\section{Demographic and clinical characteristics}

A total of 56,149 rectal cancer patients in the SEER database met our screening criteria from 2010 to 2016 (Figure 1). $56.6 \%$ of these people were younger than 65 years old, while $43.4 \%$ older than 65 years old. The ratio of females to males was about 1:1.4. Most of them were white (76.8\%) and married (73.2\%). As for the TNM stage, T3 and N0 stage accounted for the majority, $32.7 \%$ and $53.0 \%$ respectively. Among these patients, $1.4 \%, 0.2 \%$, and $5.8 \%$ were diagnosed with bone metastasis, brain metastasis, or lung metastasis, respectively. $72.2 \%$ of the patients underwent surgical treatment after diagnosis, including primary rectal lesions and/or distant metastatic lesions. The demographic and clinical data are displayed in Table 1.

\section{Incidence of liver metastases}

In the entire cohort, the prevalence of liver metastases was $11.9 \%$ $(6,662 / 56,149)$ in the initial diagnosis of rectal cancer (Table 1). And all of the 6,662 cases with liver metastases had been actively followed up, as the mean follow-up time was 15.9 months (Table 2).

\section{Risk factors for spreading liver metastases}

Univariate analysis indicated diverse elements were notably related to liver metastases occurrence. These factors were identified as age above 65 years old $(\mathrm{OR}=0.89,95 \% \mathrm{CI}$ : $0.85-0.94, \mathrm{P}<0.001)$; male $(\mathrm{OR}=0.07,95 \%$ CI: $0.73-0.82, \mathrm{P}<0.001)$; black race $(\mathrm{OR}=1.29,95 \%$ CI: 1.17-1.40, $\mathrm{P}<0.001)$; marital status (OR=1.57, 95\% CI: 1.40-1.76, $\mathrm{P}<0.001)$; presence of bone metastases $(\mathrm{OR}=15.21,95 \% \mathrm{CI}: 13.12$ 17.63, $\mathrm{P}<0.001)$, brain metastases $(\mathrm{OR}=7.18,95 \% \mathrm{CI}: 5.12-10.08$, $\mathrm{P}<0.001$ ), and lung metastases ( $\mathrm{OR}=20.65,95 \% \mathrm{CI}: 19.09-22.34$, $\mathrm{P}<0.001)$, and surgical treatments $(\mathrm{OR}=0.10,95 \% \mathrm{CI}: 0.09-0.10$, $\mathrm{P}<0.001)$.

Multivariate logistic regression analyses showed younger age, female, unmarried status, black race, advanced $\mathrm{T}$ or $\mathrm{N}$ stage, presence of bone or lung metastases, and lack of any surgery treatment were

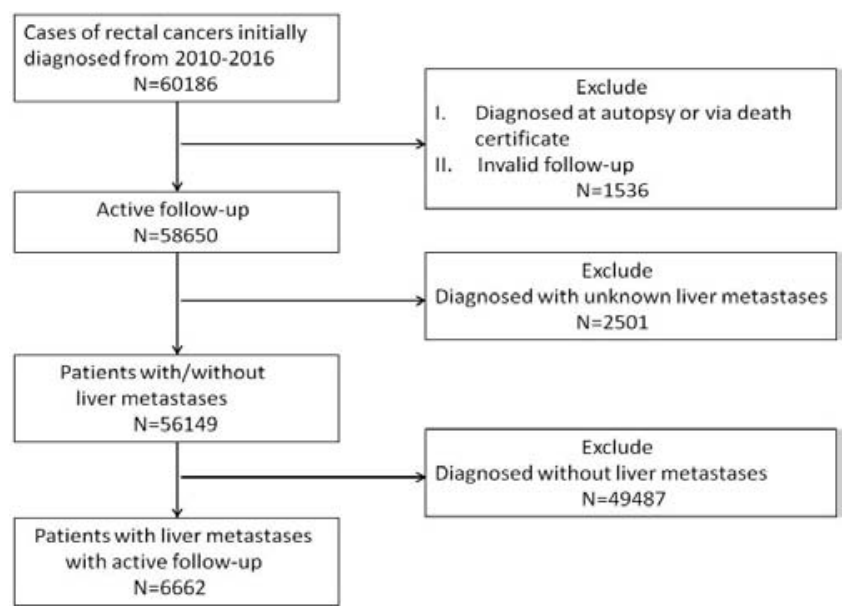

Figure 1: Flowchart of patient inclusion in this cohort study. First, "year of diagnosis" is "2010, 2011, 2012, 2013, 2014, 2015, 2016"; "primary site-labeled" is "C20.9-Rectum, NOS". 60186 rectal cancer patients were selected. Second, "type of reporting source" is not "autopsy only" and "death certificate only"; "survival months flag" is "complete datas are availabe and there are more than 0 days of survival". The number of active follow-up patients is 58650 . Third, "SEER combined mets at DX-liver (2010+)" in "extent of disease" is not "NA, unknown, blanks". The number of patients with/without liver metastases is 56149. Last, "SEER combined mets at DX-liver (2010+)" in "extent of disease" is not "no". The number of patients with liver metastases is 6662. 
Table 1: Univariate and multivariable logistic regression for analyzing the demographic and related clinical characteristics for developing liver metastases in patients diagnosed with initial rectal cancer (diagnosed 2010-2016).

\begin{tabular}{|c|c|c|c|c|c|c|}
\hline \multirow{2}{*}{ Subject characteristics } & \multicolumn{2}{|c|}{ No. of rectal cancer patients } & \multicolumn{2}{|c|}{ Univariable } & \multicolumn{2}{|c|}{ Multivariable } \\
\hline & With liver met (N, \%) & Without liver met (N, \%) & OR $(95 \% \mathrm{Cl})$ & $P$ & OR $(95 \% \mathrm{Cl})$ & $P$ \\
\hline \multicolumn{7}{|l|}{ Age } \\
\hline$<65$ & $3930(12.4)$ & 27827 (87.6) & 1 (Reference) & 1 & 1 (Reference) & 1 \\
\hline$\geq 65$ & $2732(11.2)$ & $21660(88.8)$ & $0.89(0.85-0.94)$ & $<0.001$ & $0.68(0.64-0.72)$ & $<0.001$ \\
\hline \multicolumn{7}{|l|}{ Gender } \\
\hline Female & $2415(10.3)$ & $20971(89.7)$ & 1 (Reference) & 1 & 1 (Reference) & 1 \\
\hline Male & 4247 (13.0) & $28516(87.0)$ & $0.07(0.73-0.82)$ & $<0.001$ & $0.74(0.70-0.79)$ & $<0.001$ \\
\hline \multicolumn{7}{|l|}{ Race } \\
\hline White & $5231(12.1)$ & 37892 (87.9) & 1 (Reference) & 1 & 1 (Reference) & 1 \\
\hline Black & $795(12.3)$ & $5673(87.7)$ & $1.29(1.17-1.40)$ & $<0.001$ & $1.21(1.09-1.35)$ & $<0.001$ \\
\hline Others & $636(9.7)$ & $5922(90.3)$ & NA & NA & NA & NA \\
\hline Married & 4671 (11.4) & 36446 (88.6) & 1 (Reference) & 1 & 1 (Reference) & 1 \\
\hline Unmarried & 1659 (15.6) & 8977 (84.4) & $1.57(1.40-1.76)$ & $<0.001$ & $1.77(1.55-2.21)$ & $<0.001$ \\
\hline Unknown & $332(7.6)$ & 4064 (92.4) & NA & NA & NA & NA \\
\hline \multicolumn{7}{|l|}{ T stage } \\
\hline T0 & $8(0.5)$ & 1694 (99.5) & 1 (Reference) & 1 & 1 (Reference) & 1 \\
\hline $\mathrm{T} 1$ & 788 (6.6) & 11167 (93.4) & $14.94(7.43-30.04)$ & $<0.001$ & $8.61(4.26-17.42)$ & $<0.001$ \\
\hline $\mathrm{T} 2$ & $216(3.9)$ & $5329(96.1)$ & $8.58(4.23-17.42)$ & $<0.001$ & $5.55(2.71-11.34)$ & $<0.001$ \\
\hline T3 & 1958 (10.7) & 16399 (89.3) & $25.28(12.60-50.72)$ & $<0.001$ & $9.63(4.77-19.45)$ & $<0.001$ \\
\hline $\mathrm{T} 4$ & $781(20.1)$ & 3107 (79.9) & $53.23(26.45-107.08)$ & $<0.001$ & $11.68(5.76-23.70)$ & $<0.001$ \\
\hline Unknown & $2911(19.8)$ & $11791(80.2)$ & NA & NA & NA & NA \\
\hline \multicolumn{7}{|l|}{ N stage } \\
\hline $\mathrm{N} 1$ & $2101(17.4)$ & 9958 (82.6) & $2.85(2.67-3.04)$ & $<0.001$ & $2.44(2.24-2.65)$ & 0.005 \\
\hline N2 & $624(17.7)$ & 2895 (82.3) & $2.91(2.64-3.21)$ & $<0.001$ & $3.99(3.55-4.51)$ & $<0.001$ \\
\hline Unknown & $1884(17.5)$ & $8900(82.5)$ & NA & NA & NA & NA \\
\hline \multicolumn{7}{|l|}{ Bone Met } \\
\hline None & $5958(10.8)$ & $49160(89.2)$ & 1 (Reference) & 1 & 1 (Reference) & 1 \\
\hline Yes & $516(64.8)$ & $280(35.2)$ & $15.21(13.12-17.63)$ & $<0.001$ & $3.36(2.82-4.01)$ & $<0.001$ \\
\hline Unknown & $188(80.0)$ & $47(20.0)$ & NA & NA & NA & NA \\
\hline \multicolumn{7}{|l|}{ Brain Met } \\
\hline None & $6383(11.4)$ & $49376(88.6)$ & 1 (Reference) & 1 & 1 (Reference) & 1 \\
\hline Yes & $65(48.1)$ & 70 (51.9) & $7.18(5.12-10.08)$ & $<0.001$ & $0.95(0.63-1.44)$ & 0.815 \\
\hline Unknown & $214(83.9)$ & $41(16.1)$ & NA & NA & NA & NA \\
\hline \multicolumn{7}{|l|}{ Lung Met } \\
\hline None & 4359 (8.3) & 48253 (91.7) & 1 (Reference) & 1 & 1 (Reference) & 1 \\
\hline Yes & $2121(65.1)$ & 1137 (34.9) & $20.65(19.09-22.34)$ & $<0.001$ & $7.71(7.05-8.42)$ & $<0.001$ \\
\hline Unknown & $182(65.2)$ & $97(34.8)$ & NA & NA & NA & NA \\
\hline \multicolumn{7}{|l|}{ Surg } \\
\hline None & 4707 (32.6) & $9748(67.4)$ & 1 (Reference) & 1 & 1 (Reference) & 1 \\
\hline Yes & $1799(4.4)$ & 38745 (95.6) & $0.10(0.09-0.10)$ & $<0.001$ & $0.14(0.13-0.15)$ & $<0.001$ \\
\hline Unknown & $156(13.6)$ & $994(86.4)$ & NA & NA & NA & NA \\
\hline
\end{tabular}

Notes: All factors with unknown data were removed from the Cox and Kaplan-Meier model.

Abbreviations: IQR: Interquartile Range; Met: Metastases; Surg: Surgical Treatments; NA: Not Available. 

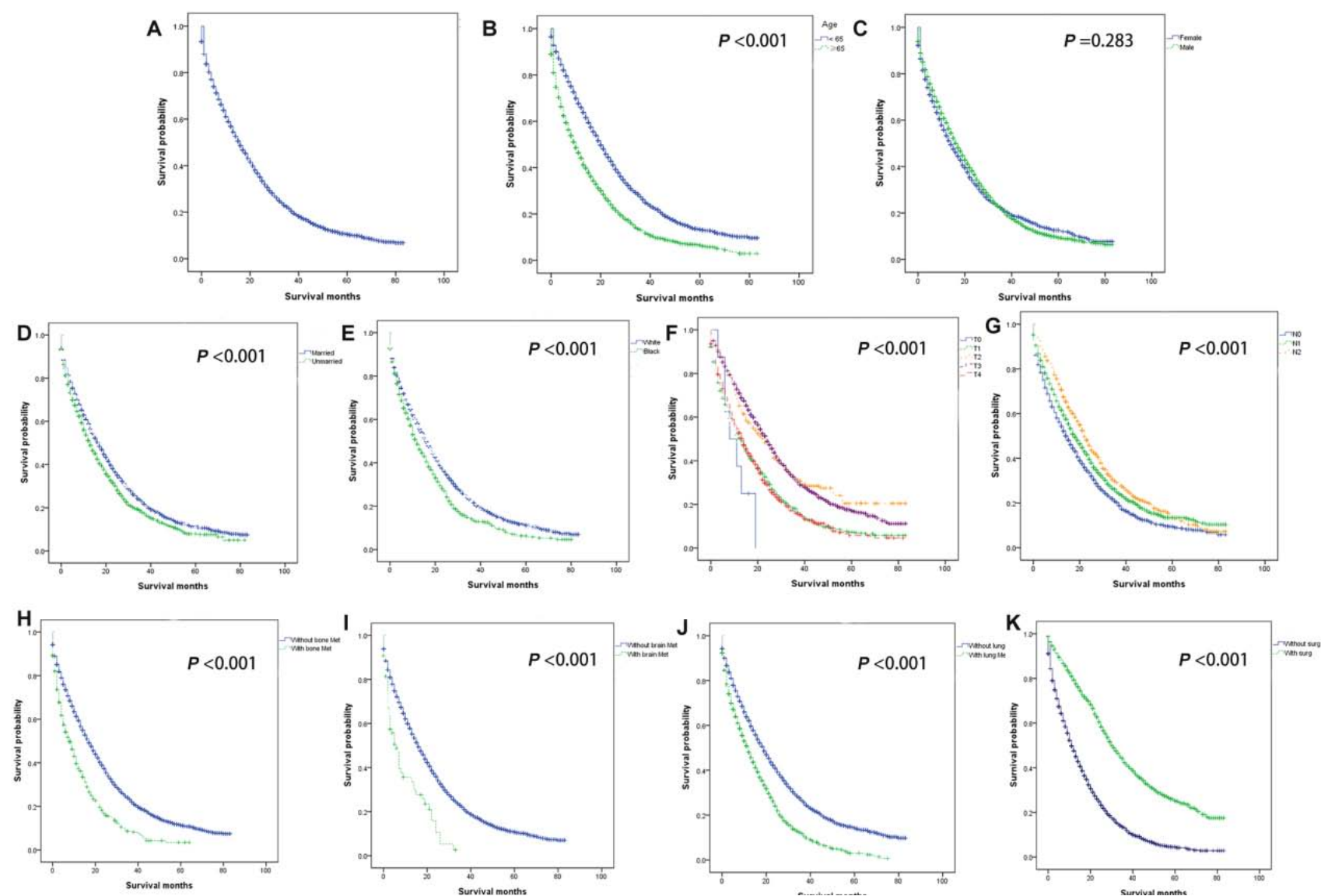

Figure 2: Kaplan-Meier analysis of overall survival among patients diagnosed with rectal cancer with initial liver metastases (A, overall), stratifed by age (B), gender $(C)$, marital status $(D)$, race $(E), T$ stage $(F), N$ stage $(G)$, bone metastases $(H)$, brain metastases $(I)$, lung metastases $(J)$, and surgical treatments $(K)$.

positively related to liver metastases at initial diagnosis (Table 1).

\section{Survival analysis and prognostic factors for liver metastases}

$69.7 \%(\mathrm{~N}=4644)$ of rectum cancer patients with initial liver metastases died before the follow-up ended. The median OS for these rectum cancer patients was 16.0 months (95\% CI: $15.34-16.66$ months, Figure 2A). Older age (Figure 2B), unmarried status (Figure $2 \mathrm{D}$ ), Black race (Figure 2E) and the complication of bone metastases (Figure 2H), brain metastases (Figure 2I), or lung metastases (Figure $2 \mathrm{~J})$ were negatively related to OS by univariate analysis model. In contrast, it was found that surgical treatment was directly related to OS $(\mathrm{P}<0.001$, Figure $2 \mathrm{~K})$.

The OS of patients with elder age $(\mathrm{HR}=1.68,95 \% \mathrm{CI}$ : 1.59 1.79, $\mathrm{P}<0.001)$, Black race $(\mathrm{HR}=1.23,95 \% \mathrm{CI}: 1.13-1.34, \mathrm{P}<0.001)$, unmarried status $(\mathrm{HR}=1.26,95 \% \mathrm{CI}: 1.18-1.34, \mathrm{P}<0.001)$, bone metastases ( $\mathrm{HR}=1.41,95 \% \mathrm{CI}: 1.27-1.57, \mathrm{P}<0.001)$, brain metastases $(\mathrm{HR}=1.63,95 \% \mathrm{CI}: 1.24-2.15, \mathrm{P}<0.001)$, and lung metastases ( $\mathrm{HR}=1.26,95 \%$ CI: $1.18-1.34, \mathrm{P}<0.001)$ was poorer than the control groups according to the multivariate Cox regression. The OS of patients in the surgical group obviously exceeded that of the nonoperative group. $(\mathrm{HR}=0.47,95 \% \mathrm{CI}: 0.43-0.51, \mathrm{P}<0.001)$ ). The median survival time of the patients in the surgery group was 30 months, while the unoperated group was 11 months (Table 2).

\section{Discussion}

To our knowledge, this is a population-based study of liver metastases in rectal cancer to detect the prevalence, hazard and forecast elements. Given the deleterious effects of liver metastases on survival in rectal cancer patients, screening methods to determine whether a rectal cancer patient is subjected to liver metastases should be recommended. The results of our study indicate that patients with age below 65 years old, female, black, unmarried, advanced $\mathrm{T}$ stage, advanced $\mathrm{N}$ stage, or bone, brain, or lung metastases showed significantly higher chance of liver metastases at diagnosis of rectal cancer. Therefore, liver ultrasound or MRI in the initial diagnosis of rectal cancer patients should be promising and deserve to be taken into considerations in patients with the above factors.

Previous studies have reported various prognostic factors for rectal cancer, including serum CEA $[9,10]$, radiation therapy [11], circumferential resection margin [12], and marital status [13]. By using the SEER database, we discovered seven prognostic elements for liver metastases in rectal cancer patients at initial diagnosis. These patients had a notably higher risk of mortality if they were older than 65 years, black, unmarried, with bone, brain or lung metastases, or no surgery. It is better to understand that older patients have a worse prognosis. It is not hard to find elder patients are more likely to have poorer physical foundations, more comorbidities, and poorer 
Table 2: Multivariable Cox regression for analyzing the prognosis factors for primary rectal cancer with liver metastases.

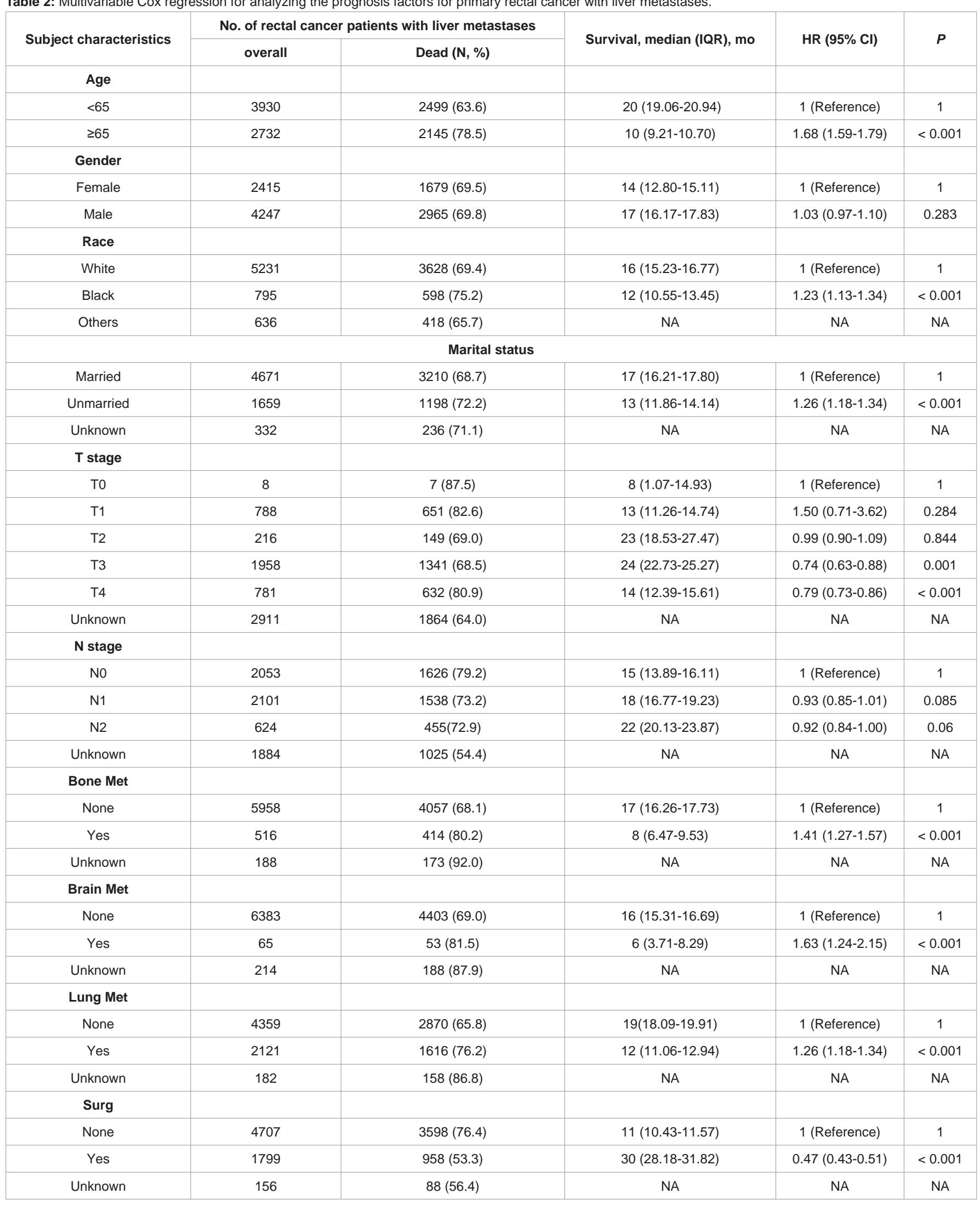

Notes: All factors with unknown data were removed from the Cox and Kaplan-Meier model.

Abbreviations: IQR: Interquartile Range; Met: Metastases; Surg: Surgical Treatments; NA: Not Available. 
tolerance to treatment [14]. The association between ethnicity and mortality is not assured and contributed by a variety of factors. Firstly, the prognosis of the black race is worse than that of the white race, which may be partly related to ethnic genes [15]. Secondly, different economic conditions of different races also give rise to the conclusions to certain extent. Thirdly, different education background also brings out different attention paid by the patients to their health status [16]. Meanwhile there are reasons for the correlation between marital status and mortality. The poor survival outcome of unmarried patients may lack social and family care and support while the spouse of a married patient will encourage him/her to receive anti-tumor treatment. Moreover, married patients may show less pain and low mood after diagnosed cancer. They can obtain spouse support which allows them to receive treatment and ensure medication compliance with better mental status $[17,18]$. Patients with other distant organ metastases, including bone, brain, or lung metastases, have a worse prognosis. This is found in our research, and it is consistent with other findings [19]. A possible explanation could be related to the high invasiveness of tumor cells but the mechanisms behind these phenomena require to be further explored.

However, the firstly perform primary site surgery or first remove liver lesions is remaining controversial for rectal cancer liver metastases patients, even though these patients have generally been considered suitable for surgical resection with a healing intent [4]. In this research, a notably raising survival rate was inspected in patients who had received surgery. However, due to the incomplete data, we are unable to know where the tumors have been removed from these patients. As the data become further detailed, we hope to analyse the specific benefits of surgery for rectal cancer liver metastases patients combined with bone, brain or lung metastases.

An interesting phenomenon has also been found in our analysis. In the Kaplan-Meier analysis of OS, it was not the advanced $\mathrm{T}$ or $\mathrm{N}$ stage patients whose prognosis was worse. This means that the evaluation of prognosis for the grades of $\mathrm{T}$ and $\mathrm{N}$ in rectal cancer patients who have developed distant organs is not of majority importance. In particular, we have seen patients with T0 stage, who were unable to find primary lesions in imaging, but their prognosis was the worst. It may show that the tumor cells of these patients are highly invasive, and distant metastases are performed at an early stage. The high degree of invasiveness also indicates the poor overall prognosis of these patients. This was consistent with others study [20]. The specific molecular biological mechanism needs to be further explored.

There is no denying that our research has some limitations. First, the study was a retrospective analysis with classical bias. In the future analysis, randomized controlled clinical trials are necessary. Second, the data were acquired from the SEER database, which cannot be representative of the global population. Third, due to the limitations of the data, detailed treatment options, including the specific site of surgery, chemotherapy regimen, and radiation therapy dose, are not available, which hamper further prognostic analysis given the detailed treatment protocols.

\section{Conclusions}

In patients with newly diagnosed rectal cancer, the incidence of liver metastases was close to $11 \%$. Liver metastasis significantly reduced the survival rate of rectal cancer patients. This gives rise to the proposal that ultrasound or MRI examinations of the liver should be considered as a routine examination of young, unmarried, female, and black ethnic rectal cancer patients at the time of initial diagnosis. Our data identify the risk and prognostic factors of liver metastasis rectal cancer patients, which might provide basic evidence for early detection of liver metastases and advanced indications for clinicians to optimize individualized diagnosis and treatment plans which will promote the patient's survival.

\section{Declarations}

\section{Ethics approval and consent to participate}

Data dissemination in the SEER database does not require the ethics approval and consent to participate. Our research is consistent with the Helsinki Declaration and related ethical norms.

\section{Consent for publication}

Data dissemination in the SEER database does not require the consent from patients.

\section{Availability of data and materials}

The datasets generated during the current study are available in the SEER program database between 2010 and 2016.

\section{Funding}

Xiaojing Yang is the recipient of a grant from Shanghai Jiao Tong University Affiliated Sixth People's Hospital (contract grant number: ynlc201807) and a grant from Shanghai Health and Family Planning Commission (20184Y0229). Jie Fu is the recipient of a grant of Shanghai Shenkang Hospital Development Center Funds for Three Year Action Plan for Promoting Clinical Skills and Clinical Innovation Capacity in Municipal Hospitals (16CR3112B). Xiaojing Yang conceived, designed, wrote and edited the manuscript. JF reviewed and edited the manuscript.

\section{Authors' Contributions}

XJY, HR, YHS, YS, LZ, HL, XZ, and XMY conceived and designed the study. XJY and HR wrote the manuscript. XJY and JF reviewed and edited the manuscript. All authors have read and approved the manuscript.

\section{References}

1. Canto L, Cury SS, Barros-Filho MC, et al. Locally advanced rectal cancer transcriptomic-based secretome analysis reveals novel biomarkers useful to identify patients according to neoadjuvant chemoradiotherapy response. Sci Rep. 2019; 9: 8702

2. Adam R, De Gramont A, Figueras J, et al. Managing synchronous liver metastases from colorectal cancer: a multidisciplinary international consensus. Cancer Treat Rev. 2015; 41: 729-741.

3. Sager O, Dincoglan F, Demiral S, et al. A Concise Review of Pelvic Radiation Therapy (RT) for Rectal Cancer with Synchronous Liver Metastases. Int J Surg Oncol. 2019; 2019: 5239042

4. Conrad C, Vauthey JN, Masayuki O, et al. Individualized Treatment Sequencing Selection Contributes to Optimized Survival in Patients with Rectal Cancer and Synchronous Liver Metastases. Ann Surg Oncol. 2017; 24: 3857-3864.

5. Ironside N, Bell R, Bartlett A, et al. Systematic review of perioperative and survival outcomes of liver resections with and without preoperative portal vein embolization for colorectal metastases. HPB (Oxford). 2017; 19: 559-566. 
6. Wang Z, Wang X, Zhang Z, et al. Association between Primary Tumo Location and Prognostic Survival in Synchronous Colorectal Liver Metastases after Surgical Treatment: A Retrospective Analysis of SEER Data. J Cancer. 2019; 10: 1593-1600.

7. Frattini M, Balestra D, Suardi S, et al. A. Different genetic features associated with colon and rectal carcinogenesis. Clin Cancer Res. 2004; 10: 4015-4021.

8. Berger MD, Yang D, Sunakawa Y, et al. Impact of sex, age, and ethnicity/race on the survival of patients with rectal cancer in the United States from 1988 to 2012. Oncotarget. 2016; 7: 53668-53678.

9. Tan Y, Fu D, Li D, et al. Predictors and Risk Factors of Pathologic Complete Response Following Neoadjuvant Chemoradiotherapy for Rectal Cancer: A Population-Based Analysis. Front Oncol. 2019; 9: 497.

10. Liu Q, Lian $P$, Luo D, et al. Combination of carcinoembryonic antigen with the American Joint Committee on Cancer TNM staging system in rectal cancer: a real-world and large population-based study. Onco Targets Ther. 2018; 11 5827-5834.

11. Liu Q, Shan Z, Luo D, et al. Palliative beam radiotherapy offered real-world survival benefit to metastatic rectal cancer: A large US population-based and propensity score-matched study. J Cancer . 2019; 10: 1216-1225.

12. Liu Q, Luo D, Cai S, et al. Circumferential resection margin as a prognostic factor after rectal cancer surgery: A large population-based retrospective study. Cancer Med. 2018; 7: 3673-3681.

13. Wang X, Cao W, Zheng C, et al. Marital status and survival in patients with rectal cancer: An analysis of the Surveillance, Epidemiology and End Results (SEER) database. Cancer Epidemiol. 2018; 54: 119-124.
14. Keevil VL, Luben R, Hayat S, et al. Physical capability predicts mortality in late mid-life as well as in old age: Findings from a large British cohort study. Arch Gerontol Geriatr. 2018; 74: 77-82.

15. Bhopal RS, Gruer L, Cezard G, et al. Mortality, ethnicity, and country of birth on a national scale, 2001-2013: A retrospective cohort (Scottish Health and Ethnicity Linkage Study). PLoS Med. 2018; 15: e1002515.

16. Ko Y, Chee W, Im EO. Factors Associated With Perceived Health Status of Multiracial/Ethnic Midlife Women in the United States. J Obstet Gynecol Neonatal Nurs. 2016; 4: 378-390.

17. Haley WE. Family caregivers of elderly patients with cancer: understanding and minimizing the burden of care. J Support Oncol. 2003; 1: 25-29.

18. Aizer AA, Paly JJ, Zietman AL, et al. Multidisciplinary care and pursuit of active surveillance in low-risk prostate cancer. J Clin Oncol. 2012; 30: 30713076.

19. Luo D, Liu Q, Yu W, et al. Prognostic value of distant metastasis sites and surgery in stage IV colorectal cancer: a population-based study. Int J Colorectal Dis. 2018; 33 1241-1249.

20. Magni E, Botteri E, Ravenda PS, et al. Detection of circulating tumor cells in patients with locally advanced rectal cancer undergoing neoadjuvant therapy followed by curative surgery. Int J Colorectal Dis. 2014; 29: 1053-1059. 